\title{
A NEW CLASS OF MEROMORPHIC FUNCTIONS USING D OPERATOR
}

\section{Dr. Deepaly Nigam}

Abstract: We introduce a new class $\mathrm{M}_{\mathrm{p}}^{\mathrm{k}}(m, \alpha)$ of functions of the form $\mathrm{f}(\mathrm{z})=\frac{1}{\mathrm{z}^{\mathrm{p}}}+\sum_{\mathrm{n}=0}^{\infty} \mathrm{a}_{\mathrm{n}+\mathrm{k}} \mathrm{z}^{\mathrm{n}+\mathrm{k}}, \mathrm{a}_{\mathrm{n}+\mathrm{k}} \geq 0, k \in N_{0}$ which are regular in the punctured unit disk $U^{*}=$

$\{Z: Z \in \not \subset: 0<|Z|<1\}$. Sharp results concerning coefficients, distortion, closure properties, integral operator, neighborhood property, inclusion property and radii of starlikeness and convexity for the class are determined.

\section{Introduction}

Let $\mathrm{M}_{\mathrm{p}}^{\mathrm{k}}$ denote the class of functions of the form

$$
f(z)=\frac{1}{z^{p}}+\sum_{n=0}^{\infty} a_{n+k} z^{n+k} ; \quad a_{n+k} \geq 0, p \in N \text { and } k \in N_{0} .
$$

which are analytic in puntured unit disk $U^{*}=\{Z: Z \in \not \subset: 0<|Z|<1\}$. We define the differential operator $D^{m}$ on functions belonging to the class $\mathrm{M}_{\mathrm{p}}^{\mathrm{k}}$, using convolution as follows :

$$
\mathrm{D}^{\mathrm{m}} \mathrm{f}(\mathrm{z})=\frac{1}{\mathrm{z}^{\mathrm{p}}(1-\mathrm{z})^{\mathrm{m}+1}} * \mathrm{f}(\mathrm{z}) \quad \mathrm{m} \in \mathrm{N}_{0}=\{0,1,2, \ldots\}
$$

or

Clearly,

$$
\begin{aligned}
& D^{m} f(z)=\frac{1}{z^{p} m ! d z^{m}} \quad\left(z^{m+p} f(z)\right) \\
& D^{m} f(z)=\frac{1}{z^{p}}+\sum_{n=0}^{\infty} \frac{(m+p+n+k) !}{m !(n+p+k) !} a_{n+k} z^{n+k}
\end{aligned}
$$

$\mathrm{D}^{0} \mathrm{f}(\mathrm{z})=\mathrm{f}(\mathrm{z})$.

A function belonging to the class $M_{p}^{k}$ is said to be meromorphically starlike of order ' $\alpha$ ' if and only if

$$
\operatorname{Re}\left(-\frac{\mathrm{zf}^{\prime}(\mathrm{z})}{\mathrm{f}(\mathrm{z})}\right)>\alpha \quad ; \quad(\mathrm{z} \in \mathrm{D}, 0 \leq \alpha<\mathrm{p})
$$

The subclass of $\mathrm{M}_{\mathrm{p}}^{\mathrm{k}}$ consisting of functions which are meromorphically starlike of order $\alpha$ is denoted by $\mathrm{S}_{\mathrm{p}}^{\mathrm{k}}(\alpha)$.

We define a class $M_{p}^{k}(m, \alpha)$ of function of the form (1.1) which satisfies the condition

$$
\operatorname{Re}\left\{\frac{\mathrm{D}^{\mathrm{m}+1} \mathrm{f}(\mathrm{z})}{\mathrm{D}^{\mathrm{m}} \mathrm{f}(\mathrm{z})}\right\}<\frac{\mathrm{m}+1+\mathrm{p}-\alpha}{\mathrm{m}+1}
$$

where $\mathrm{m} \in \mathrm{N}_{0}, \mathrm{p} \in \mathrm{N}=\{1,2, \ldots\}$ and $0 \leq \alpha<\mathrm{p}$.

Clearly,

$$
M_{p}^{\mathrm{k}}(0, \alpha) \equiv \mathrm{S}_{\mathrm{p}}^{\mathrm{k}}(\alpha)
$$


Many important properties of certain subclasses of meromorphic $\mathrm{p}$-valent functions were studied by several authors including Aouf and Srivastava [1], Joshi and Srivastava [2], Liu and Srivastava [3], Liu and Owa [4], Liu and Srivastava [5], Owa et.al. [6], and Srivastava et.al. [7].

Extending the work of Liu and Owa [5] we obtain the coefficient inequality, inclusion property, distortion theorems, integral operator, neighbourhood properties, closure properties and radii of starlikeness and convexity for the class $M_{p}^{k}(m, \alpha)$.

\section{Inclusion Property Of The Class $M_{p}^{k}(m, \alpha)$ :}

For proving the inclusion property, we first prove the following lemma :

Lemma 2.1 : Let $f(z) \in M_{p}^{k}$ be given by (1.1), then

$$
\mathrm{z}\left(\mathrm{D}^{\mathrm{m}} \mathrm{f}(\mathrm{z})\right)^{\prime}=(\mathrm{m}+1) \mathrm{D}^{\mathrm{m}+1} \mathrm{f}(\mathrm{z})-(\mathrm{m}+\mathrm{p}+1) \mathrm{D}^{\mathrm{m}} \mathrm{f}(\mathrm{z})
$$

Proof : In view of (1.4), we have

Consider

$$
\mathrm{D}^{\mathrm{m}} \mathrm{f}(\mathrm{z})=\frac{1}{\mathrm{z}^{\mathrm{p}}}+\sum_{\mathrm{n}=0}^{\infty} \frac{(\mathrm{m}+\mathrm{p}+\mathrm{n}+\mathrm{k}) !}{\mathrm{m} !(\mathrm{n}+\mathrm{p}+\mathrm{k}) !} \mathrm{a}_{\mathrm{n}+\mathrm{k}} \mathrm{z}^{\mathrm{n}+\mathrm{k}}
$$

$$
\begin{aligned}
(\mathrm{m}+1) \mathrm{D}^{\mathrm{m}} \mathrm{f}(\mathrm{z}) & -(\mathrm{m}+\mathrm{p}+1) \mathrm{f}(\mathrm{z})=\frac{(\mathrm{m}+1)}{\mathrm{z}^{\mathrm{p}}}+\sum_{\mathrm{n}=0}^{\infty} \frac{(\mathrm{m}+1+\mathrm{p}+\mathrm{n}+\mathrm{k}) !}{(\mathrm{m}+1) !(\mathrm{n}+\mathrm{p}+\mathrm{k}) !} \mathrm{a}_{\mathrm{n}+\mathrm{k}} \mathrm{z}^{\mathrm{n}+\mathrm{k}} \\
& -\frac{(\mathrm{m}+\mathrm{p}+1)}{\mathrm{z}^{\mathrm{p}}}-\sum_{\mathrm{n}=0}^{\infty} \frac{(\mathrm{m}+\mathrm{p}+\mathrm{n}+\mathrm{k}) !}{(\mathrm{m}+1) !(\mathrm{n}+\mathrm{p}+\mathrm{k}) !} \mathrm{a}_{\mathrm{n}+\mathrm{k}} \mathrm{z}^{\mathrm{n}+\mathrm{k}} \\
& =-\frac{\mathrm{p}}{\mathrm{z}^{\mathrm{p}}}+\sum_{\mathrm{n}=0}^{\infty} \frac{(\mathrm{m}+\mathrm{p}+\mathrm{n}+\mathrm{k}) !}{\mathrm{m} !(\mathrm{n}+\mathrm{p}+\mathrm{k}) !}(\mathrm{n}+\mathrm{k}) \mathrm{a}_{\mathrm{n}+\mathrm{k}} \mathrm{z}^{\mathrm{n}+\mathrm{k}} \\
& =\mathrm{z}\left(\mathrm{D}^{\mathrm{m}} \mathrm{f}(\mathrm{z})\right)^{\prime}
\end{aligned}
$$

This completes the proof of lemma.

Theorem 2.2: $\mathrm{M}_{\mathrm{p}}^{\mathrm{k}}(\mathrm{m}+1, \alpha) \subset \mathrm{M}_{\mathrm{p}}^{\mathrm{k}}(\mathrm{m}, \alpha)$ for each $\mathrm{m} \in \mathrm{N}_{0}\{0,1,2, \ldots\}$

Proof : Let $\mathrm{f}(\mathrm{z}) \in \mathrm{M}_{\mathrm{p}}^{\mathrm{k}}(\mathrm{m}+1, \alpha)$, then

$$
\operatorname{Re}\left\{\frac{D^{m+2} f(z)}{D^{m+1} f(z)}\right\}<\frac{m+2+p-\alpha}{m+2} \quad,|z|<1
$$

Define a regular function $\mathrm{w}(\mathrm{z})$ in $\mathrm{U}=\mathrm{U}^{*} \mathrm{U}\{0\}$ by

$$
\operatorname{Re}\left\{\frac{\mathrm{D}^{\mathrm{m}+1} \mathrm{f}(\mathrm{z})}{\mathrm{D}^{\mathrm{m}} \mathrm{f}(\mathrm{z})}\right\}=\frac{(\mathrm{m}+1)+(\mathrm{m}+1+2 \mathrm{p}-2 \alpha) \mathrm{w}(\mathrm{z})}{(1+\mathrm{m})(1+\mathrm{w}(\mathrm{z}))}
$$

Clearly $\mathrm{w}(0)=0$.

Logarithmic differentiation of (2.3) yields

$$
\frac{\mathrm{z}\left(\mathrm{D}^{\mathrm{m}+1} \mathrm{f}(\mathrm{z})\right)^{\prime}}{\mathrm{D}^{\mathrm{m}+1} \mathrm{f}(\mathrm{z})}-\frac{\mathrm{z}\left(\mathrm{D}^{\mathrm{m}} \mathrm{f}(\mathrm{z})\right)^{\prime}}{\mathrm{D}^{\mathrm{m}} \mathrm{f}(\mathrm{z})}=\frac{\mathrm{z}(\mathrm{m}+1+2 \mathrm{p}-2 \alpha) \mathrm{w}^{\prime}(\mathrm{z})}{(\mathrm{m}+1)+(\mathrm{m}+1+2 \mathrm{p}-2 \alpha) \mathrm{w}(\mathrm{z})}-\frac{\mathrm{zw}^{\prime}(\mathrm{z})}{1+\mathrm{w}(\mathrm{z})}
$$

Using lemma (2.1), equation (2.4) reduces to

$$
\begin{array}{r}
(\mathrm{m}+2) \frac{\mathrm{D}^{\mathrm{m}+2} \mathrm{f}(\mathrm{z})}{\mathrm{D}^{\mathrm{m}+1} \mathrm{f}(\mathrm{z})}-(\mathrm{m}+2+\mathrm{p}-\alpha)=\frac{2(\mathrm{p}-\alpha) \mathrm{w}(\mathrm{z})}{1+\mathrm{w}(\mathrm{z})}-(\mathrm{p}-\alpha) \\
\quad+\frac{2(\mathrm{p}-\alpha) \mathrm{zw}^{\prime}(\mathrm{z})}{\{(\mathrm{m}+1)+(\mathrm{m}+1+2 \mathrm{p}-2 \alpha)+\mathrm{w}(\mathrm{z})\}(1+\mathrm{w}(\mathrm{z}))}
\end{array}
$$

or

$$
\frac{\frac{\mathrm{D}^{\mathrm{m}+2} \mathrm{f}(\mathrm{z})}{\mathrm{D}^{\mathrm{m}+1} \mathrm{f}(\mathrm{z})}-\frac{(\mathrm{m}+2+\mathrm{p}-\alpha)}{\mathrm{m}+2}}{(\mathrm{p}-\alpha) /(\mathrm{m}+2)}=\frac{2 \mathrm{w}(\mathrm{z})}{1+\mathrm{w}(\mathrm{z})}-1
$$




$$
+\frac{2 \mathrm{zw}^{\prime}(\mathrm{z})}{\{(\mathrm{m}+1)+(\mathrm{m}+1+2 \mathrm{p}-2 \alpha)+\mathrm{w}(\mathrm{z})\}(1+\mathrm{w}(\mathrm{z}))}
$$

or

$$
\begin{aligned}
& \frac{\mathrm{D}^{\mathrm{m}+2} \mathrm{f}(\mathrm{z}) / \mathrm{D}^{\mathrm{m}+1} \mathrm{f}(\mathrm{z})-(\mathrm{m}+2+\mathrm{p}-\alpha) / \mathrm{m}+2}{(\mathrm{p}-\alpha) /(\mathrm{m}+2)}=\frac{-1+\mathrm{w}(\mathrm{z})}{1+\mathrm{w}(\mathrm{z})} \\
&+\frac{2 \mathrm{zw}^{1}(\mathrm{z})}{[(\mathrm{m}+1)+(\mathrm{m}+1+2 \mathrm{p}-2 \alpha)+\mathrm{w}(\mathrm{z})](1+\mathrm{w}(\mathrm{z}))}
\end{aligned}
$$

Suppose there exists a point $\mathrm{z}_{0}$ in $|\mathrm{z}|<1$ such that $\max _{\left.|\mathrm{z}|<\mid<\mathrm{z}_{0}\right\}}|\mathrm{w}(\mathrm{z})|=\left|\mathrm{w}\left(\mathrm{z}_{0}\right)\right|=1$. From a well known result due to Jack (1971), there is a real number $\mathrm{K} \geq 1$ such that

$$
\mathrm{z}_{0} \mathrm{w}^{\prime}\left(\mathrm{z}_{0}\right)=\mathrm{Kw}\left(\mathrm{z}_{0}\right)
$$

From (2.5) and (2.6), we obtain

$$
\begin{aligned}
& \frac{\frac{\mathrm{D}^{\mathrm{m}+2} \mathrm{f}\left(\mathrm{z}_{0}\right)}{\mathrm{D}^{\mathrm{m}+1} \mathrm{f}\left(\mathrm{z}_{0}\right)}-\frac{(\mathrm{m}+2+\mathrm{p}-\alpha)}{\mathrm{m}+2}}{(\mathrm{p}-\alpha) /(\mathrm{m}+2)}=\frac{-1+\mathrm{w}\left(\mathrm{z}_{0}\right)}{1+\mathrm{w}\left(\mathrm{z}_{0}\right)} \\
& +\frac{2 \mathrm{Kw}\left(\mathrm{z}_{0}\right)}{\left[(\mathrm{m}+1)+(\mathrm{m}+1+2 \mathrm{p}-2 \alpha)+\mathrm{w}\left(\mathrm{z}_{0}\right)\right]\left(1+\mathrm{w}\left(\mathrm{z}_{0}\right)\right)}
\end{aligned}
$$

Thus,

$$
\operatorname{Re}\left\{\frac{\frac{\mathrm{D}^{\mathrm{m}+2} \mathrm{f}\left(\mathrm{z}_{0}\right)}{\mathrm{D}^{\mathrm{m}+1} \mathrm{f}\left(\mathrm{z}_{0}\right)}-(\mathrm{m}+2+\mathrm{p}-\alpha)}{(\mathrm{p}-\alpha) /(\mathrm{m}+2)}\right\}>\frac{1}{2(\mathrm{~m}+1+\mathrm{p}-\alpha)}>0
$$

which contradicts (2.2). Hence, $|\mathrm{w}(\mathrm{z})|<1$ for $\mathrm{z} \in \mathrm{U}$ and from (2.3), it follows that $\mathrm{f}(\mathrm{z}) \in \mathrm{M}_{\mathrm{p}}^{\mathrm{k}}(\mathrm{m}, \alpha)$.

Therefore, $M_{\mathrm{p}}^{\mathrm{k}}(\mathrm{m}+1, \alpha) \subset \mathrm{M}_{\mathrm{p}}^{\mathrm{k}}(\mathrm{m}, \alpha)$

This completes the proof of theorem.

\section{Coefficient Inequality}

Theorem 3.1 : Let $\mathrm{f}(\mathrm{z}) \in \mathrm{M}_{\mathrm{p}}^{\mathrm{k}}$ be given by (1.1), then $\mathrm{f}(\mathrm{z}) \in \mathrm{M}_{\mathrm{p}}^{\mathrm{k}}(\mathrm{m}, \alpha)$ if

$$
\sum_{n=0}^{\infty} \frac{(m+p+n+k) !}{m !(n+p+k) !}\{(n+p+k)+|n+k-p+2 \alpha|\} a_{n+k} \leq 2(p-\alpha)
$$

for $0 \leq \alpha<\mathrm{p}$.

Proof : For $0 \leq \alpha<\mathrm{p}$, consider the expression

$$
\mathrm{H}(\mathrm{z})=\left|-\mathrm{D}^{\mathrm{m}+1} \mathrm{f}(\mathrm{z})+\mathrm{D}^{\mathrm{m}} \mathrm{f}(\mathrm{z})\right|-\left|-\mathrm{D}^{\mathrm{m}+1} \mathrm{f}(\mathrm{z})+\left(\frac{\mathrm{m}+1+2 \mathrm{p}-2 \alpha}{\mathrm{m}+1}\right) \mathrm{D}^{\mathrm{m}} \mathrm{f}(\mathrm{z})\right|
$$

Replacing $\mathrm{D}^{\mathrm{m}} \mathrm{f}(\mathrm{z})$ and $\mathrm{D}^{\mathrm{m}+1} \mathrm{f}(\mathrm{z})$ by their series expansions.

for $0<|z|=r<1$ we have

$$
\begin{aligned}
H(z)= & \left|-\sum_{n=0}^{\infty} \frac{(m+p+n+k) !}{m !(n+p+k) !} \frac{(n+p+k)}{(m+1)} a_{n+k} z^{n+k}\right| \\
& -\left|\frac{2(p-\alpha)}{(m+1) z^{p}}-\sum_{n=0}^{\infty} \frac{(m+p+n+k) !}{m !(n+p+k) !} \frac{(n+k-p+2 \alpha)}{(m+1)} a_{n+k} z^{n+k}\right|
\end{aligned}
$$




$$
\begin{aligned}
\mathrm{r}^{\mathrm{p}} \mathrm{H}(\mathrm{z}) & <\sum_{\mathrm{n}=0}^{\infty} \frac{(\mathrm{m}+\mathrm{p}+\mathrm{n}+\mathrm{k}) !}{\mathrm{m} !(\mathrm{n}+\mathrm{k}+\mathrm{p}) !} \frac{(\mathrm{p}+\mathrm{n}+\mathrm{k})}{(\mathrm{m}+1)} \mathrm{a}_{\mathrm{n}+\mathrm{k}} \mathrm{r}^{\mathrm{n}+\mathrm{k}-\mathrm{p}}-\frac{2(\mathrm{p}-\alpha)}{(\mathrm{m}+1)} \\
& +\sum_{\mathrm{n}=0}^{\infty} \frac{(\mathrm{m}+\mathrm{p}+\mathrm{n}+\mathrm{k}) !}{\mathrm{m} !(\mathrm{n}+\mathrm{k}+\mathrm{p}) !} \frac{\mathrm{n}+\mathrm{k}-\mathrm{p}+2 \alpha \mid}{(\mathrm{m}+1)} \mathrm{a}_{\mathrm{n}+\mathrm{k}} \mathrm{r}^{\mathrm{n}+\mathrm{k}-\mathrm{p}}
\end{aligned}
$$

Since this holds for all $r, 0<r<1$, making $r \rightarrow 1$ we have

$$
\mathrm{H}(\mathrm{z}) \leq \sum_{\substack{\mathrm{n}=0 \\ \leq 0}}^{\infty} \frac{(\mathrm{m}+\mathrm{p}+\mathrm{n}+\mathrm{k}) !}{\mathrm{m} !(\mathrm{n}+\mathrm{k}+\mathrm{p}) !}\left\{\frac{(\mathrm{p}+\mathrm{n}+\mathrm{k})|\mathrm{n}+\mathrm{k}-\mathrm{p}+2 \alpha|}{(\mathrm{m}+1)}\right\} \mathrm{a}_{\mathrm{n}+\mathrm{k}}-\frac{2(\mathrm{p}-\alpha)}{(\mathrm{m}+1)}
$$

From (3.2), we find

$$
\left|\left(-\frac{D^{m+1} f(z)}{D^{m} f(z)}+1\right)\left(-\frac{D^{m+1} f(z)}{D^{m} f(z)}+\frac{(m+1+2 p-2 \alpha)}{(m+1)}\right)^{-1}\right| \leq 1
$$

or

$$
\left|\frac{\left(-\frac{D^{m+1} f(z)}{D^{m} f(z)}+2\right)-1}{\left(-\frac{D^{m+1} f(z)}{D^{m} f(z)}+2\right)+\left(1-2\left(\frac{m+1-p+\alpha}{m+1}\right)\right)}\right| \leq 1
$$

or

$$
\operatorname{Re}\left(-\frac{\mathrm{D}^{\mathrm{m}+1} \mathrm{f}(\mathrm{z})}{\mathrm{D}^{\mathrm{m}} \mathrm{f}(\mathrm{z})}+2\right)>\frac{\mathrm{m}+1-\mathrm{p}+\alpha}{\mathrm{m}+1}
$$

or

$$
\operatorname{Re}\left(\frac{\mathrm{D}^{\mathrm{m}+1} \mathbf{f}(\mathrm{z})}{\mathrm{D}^{\mathrm{m}} \mathbf{f}(\mathrm{z})}\right)<\frac{\mathbf{m}+\mathbf{1}+\mathbf{p}-\boldsymbol{\alpha}}{\mathbf{m}+\mathbf{1}}
$$

Hence, $f(z) \in M_{p}^{k}(m, \alpha)$.

Theorem 3.2 : Let $f(z) \in M_{p}^{k}$ be given by (1.1). Then $f(z) \in M_{p}^{k}(m, \alpha)$ if and only if

$$
\sum_{n=0}^{\infty}(n+k+\alpha) \frac{(m+p+n+k) !}{m !(n+k+p) !} a_{n+k} \leq(p-\alpha), \quad a_{n+k} \geq 0
$$

for $\mathrm{p} / 2 \leq \alpha<\mathrm{p}$.

Proof : In view of theorem (3.1), it is sufficient to show the "only if" part. Let us assume that $f(z) \in$ $M_{p}^{k}(m, \alpha)$ then

$$
\operatorname{Re}\left(\frac{\mathrm{D}^{\mathrm{m}+1} \mathrm{f}(\mathrm{z})}{\mathrm{D}^{\mathrm{m}} \mathrm{f}(\mathrm{z})}\right)<\frac{\mathrm{m}+1+\mathrm{p}-\alpha}{\mathrm{m}+1} \quad, \quad \mathrm{z} \in \mathrm{U} .
$$

Replacing $\mathrm{D}^{\mathrm{m}+1} \mathrm{f}(\mathrm{z})$ and $\mathrm{D}^{\mathrm{m}} \mathrm{f}(\mathrm{z})$ by their series expansions, we have

$$
\operatorname{Re}\left\{\frac{\frac{1}{z^{p}}+\sum_{n=0}^{\infty} \frac{(m+1+p+n+k) !}{(m+1) !(n+k+p) !} a_{n+k} z^{n+k}}{\frac{1}{z^{p}}+\sum_{n=0}^{\infty} \frac{(m+p+n+k) !}{(m) !(n+k+p) !} a_{n+k} z^{n+k}}\right\} \leq \frac{m+1+p-\alpha}{m+1}
$$

when $\mathrm{z}$ is real, $\frac{\mathrm{zf}(\mathrm{z})}{\mathrm{f}(\mathrm{z})}$ is real and since $\mathrm{a}_{\mathrm{n}+\mathrm{k}} \geq 0$, making $\mathrm{z} \rightarrow 1^{-}$through positive values (3.5) becomes

$$
\frac{1+\sum_{n=0}^{\infty} \frac{(m+1+p+n+k) !}{(m+1) !(n+k+p) !} a_{n+k}}{1+\sum_{n=0}^{\infty} \frac{(m+p+n+k) !}{m !(n+k+p) !} a_{n+k}} \leq \frac{m+1+p-\alpha}{m+1}
$$


hence, we get

$$
\sum_{n=0}^{\infty} \frac{(m+p+n+k) !}{m !(n+k+p) !}(n+k+\alpha) a_{n+k} \leq p-\alpha
$$

Hence the result follows.

Remark : The result is sharp. The extremal function being

$$
\mathrm{f}_{\mathrm{n}}(\mathrm{z})=\frac{1}{\mathrm{z}^{\mathrm{p}}}+\frac{(\mathrm{p}-\alpha) \mathrm{m} !(\mathrm{n}+\mathrm{k}+\mathrm{p}) !}{(\mathrm{n}+\mathrm{k}+\alpha)(\mathrm{m}+\mathrm{n}+\mathrm{k}+\mathrm{p}) !} \mathrm{z}^{\mathrm{n}+\mathrm{k}} \quad, \quad, \mathrm{n}, \mathrm{k} \in \mathrm{N}_{0}=\{0,1, \ldots\}
$$

Corollary 3.3 : If $\mathrm{f}(\mathrm{z}) \in \mathrm{M}_{\mathrm{p}}^{\mathrm{k}}$ with $\mathrm{p}=1$ and $\mathrm{a}_{\mathrm{k}}=0$, then theorem 3.2 holds true for $0 \leq \alpha<1$.

\section{DISTORTION THEOREM}

Theorem 4.1 : If $f(z) \in M_{p}^{k}(m, \alpha)$, then

$$
\frac{1}{\mathrm{r}^{\mathrm{p}}}-\left(\frac{\mathrm{p}-\alpha}{\mathrm{k}+\alpha}\right) \mathrm{r}^{\mathrm{k}} \leq\left|\mathrm{D}^{\mathrm{m}} \mathrm{f}(\mathrm{z})\right| \leq \frac{1}{\mathrm{r}^{\mathrm{p}}}+\left(\frac{\mathrm{p}-\alpha}{\mathrm{k}+\alpha}\right) \mathrm{r}^{\mathrm{k}} \quad, \quad(\mathrm{k} \geq 1)
$$

and

$$
\frac{\mathrm{p}}{\mathrm{r}^{\mathrm{p}+1}}-\frac{(\mathrm{p}-\alpha)(\mathrm{k}+2 \alpha)}{(\mathrm{k}+\alpha)} \mathrm{r}^{\mathrm{k}-1} \leq\left|\left(\mathrm{D}^{\mathrm{m}} \mathrm{f}(\mathrm{z})\right)^{\prime}\right| \leq \frac{\mathrm{p}}{\mathrm{r}^{\mathrm{p}+1}}+\frac{(\mathrm{p}-\alpha)(\mathrm{k}+2 \alpha)}{(\mathrm{k}+\alpha)} \mathrm{r}^{\mathrm{k}-1},(\mathrm{k} \geq 2)
$$

for $\mathrm{a}<|\mathrm{z}|=\mathrm{r}<1$ and $\mathrm{p} / 2 \leq \alpha<\mathrm{p}$.

Proof : In view of theorem (3.2), we have

$$
\sum_{n=0}^{\infty} \frac{(m+p+n+k) !}{m !(n+p+k) !} a_{n+k} \leq \frac{p-\alpha}{k+\alpha}
$$

Thus, for $0<|z|=r<1$

$$
\begin{gathered}
\left|\mathrm{D}^{\mathrm{m}} \mathrm{f}(\mathrm{z})\right| \leq \frac{1}{\mathrm{r}^{\mathrm{p}}}+\mathrm{r}^{\mathrm{k}} \sum_{\mathrm{n}=0}^{\infty} \frac{(\mathrm{m}+\mathrm{p}+\mathrm{n}+\mathrm{k}) !}{\mathrm{m} !(\mathrm{n}+\mathrm{p}+\mathrm{k}) !} \mathrm{a}_{\mathrm{n}+\mathrm{k}} \\
\left|\mathrm{D}^{\mathrm{m}} \mathrm{f}(\mathrm{z})\right| \leq \frac{1}{\mathrm{r}^{\mathrm{p}}}+\frac{(\mathrm{p}-\alpha)}{(\mathrm{k}+\alpha)} \mathrm{r}^{\mathrm{k}} \quad \text { (on using 4.2) } \\
\left|\mathrm{D}^{\mathrm{m}} \mathrm{f}(\mathrm{z})\right| \geq \frac{1}{\mathrm{r}^{\mathrm{p}}}-\mathrm{r}^{\mathrm{k}} \sum_{\mathrm{n}=0}^{\infty} \frac{(\mathrm{m}+\mathrm{p}+\mathrm{n}+\mathrm{k}) !}{\mathrm{m} !(\mathrm{n}+\mathrm{p}+\mathrm{k}) !} \mathrm{a}_{\mathrm{n}+\mathrm{k}} \\
\geq \frac{1}{\mathrm{r}^{\mathrm{p}}}-\frac{(\mathrm{p}-\alpha)}{(\mathrm{k}+\alpha)} \mathrm{r}^{\mathrm{k}} \quad \text { (on using 4.2) }
\end{gathered}
$$

From (4.3) and (4.4), we get (4.1a).

Again, by theorem (3.2), we have

$$
\begin{aligned}
\sum_{n=0}^{\infty} \frac{(n+k)(m+p+n+k) !}{m !(n+p+k) !} a_{n+k} & \leq(p-\alpha)-\alpha \sum_{n=0}^{\infty} \frac{(m+p+n+k) !}{m !(n+p+k) !} a_{n+k} \\
\leq & (p-\alpha)+\alpha \sum_{n=0}^{\infty} \frac{(m+p+n+k) !}{m !(n+p+k) !} a_{n+k} \\
\sum_{n=0}^{\infty} \frac{(n+k)(m+p+n+k) !}{m !(n+p+k) !} a_{n+k} & \leq(p-\alpha)+\alpha\left(\frac{p-\alpha}{k+\alpha}\right) \quad \text { from (4.2) } \\
& =\frac{(p-\alpha)(k+2 \alpha)}{(k+\alpha)}
\end{aligned}
$$

Thus,

$$
\sum_{n=0}^{\infty}(n+k) \frac{(m+p+n+k) !}{m !(n+p+k) !} a_{n+k} \leq \frac{(p-\alpha)(k+2 \alpha)}{(k+\alpha)}
$$

Further, for using $\mathrm{k} \geq 2$ and using (4.5), we get 
and

$$
\begin{gathered}
\left|\left(\mathrm{D}^{\mathrm{m}} \mathrm{f}(\mathrm{z})\right)^{\prime}\right| \leq \frac{1}{\mathrm{r}^{\mathrm{p}+1}}-\mathrm{r}^{\mathrm{k}} \sum_{\mathrm{n}=0}^{\infty}(\mathrm{n}+\mathrm{k}) \frac{(\mathrm{m}+\mathrm{p}+\mathrm{n}+\mathrm{k}) !}{\mathrm{m} !(\mathrm{n}+\mathrm{p}+\mathrm{k}) !} \mathrm{a}_{\mathrm{n}+\mathrm{k}} \mathrm{r}^{\mathrm{n}+\mathrm{k}-1} \\
\leq \frac{\mathrm{p}}{\mathrm{r}^{\mathrm{p}+1}}+\frac{(\mathrm{p}-\alpha)(\mathrm{k}+2 \alpha)}{(\mathrm{k}+\alpha)} \mathrm{r}^{\mathrm{k}-1}
\end{gathered}
$$

and

$$
\begin{gathered}
\left|\left(D^{m} f(z)\right)^{\prime}\right| \geq \frac{p}{r^{p+1}}-r^{k-1} \sum_{n=0}^{\infty}(n+k) \frac{(m+p+n+k) !}{m !(n+p+k) !} a_{n+k} \\
\geq \frac{p}{r^{p+1}}-\frac{(p-\alpha)(k+2 \alpha)}{(k+\alpha)} r^{k-1}
\end{gathered}
$$

Using (4.6) and (4.7), we get (4.1b).

Remark : The bounds in (4.1a) and (4.1b) are sharp. Since the equalities are attained for the function

$$
\mathrm{f}(\mathrm{z})=\frac{1}{\mathrm{z}^{\mathrm{p}}}+\frac{(\mathrm{p}-\alpha) \mathrm{m} !(\mathrm{k}+\mathrm{p}) !}{(\mathrm{k}+\alpha)(\mathrm{m}+\mathrm{p}+\mathrm{k}) !} \mathrm{z}^{\mathrm{k}} \quad(\mathrm{z}= \pm \mathrm{r}) .
$$

Corollary 4.2 : If $f(z) \in M_{p}^{k}(0, \alpha) \equiv S_{p}^{k}(\alpha)$, then

$$
\frac{1}{\mathrm{r}^{\mathrm{p}}}-\left(\frac{\mathrm{p}-\alpha}{\mathrm{k}+\alpha}\right) \mathrm{r}^{\mathrm{k}} \leq|\mathrm{f}(\mathrm{z})| \leq \frac{1}{\mathrm{r}^{\mathrm{p}}}+\left(\frac{\mathrm{p}-\alpha}{\mathrm{k}+\alpha}\right) \mathrm{r}^{\mathrm{k}} \quad(\mathrm{k} \geq 1)
$$

and

$$
\frac{\mathrm{p}}{\mathrm{r}^{\mathrm{p}+1}}-\left(\frac{(\mathrm{p}-\alpha)(\mathrm{k}+2 \alpha)}{(\mathrm{k}+\alpha)}\right) \mathrm{r}^{\mathrm{k}-1} \leq\left|\mathrm{f}^{\prime}(\mathrm{z})\right| \leq \frac{\mathrm{p}}{\mathrm{r}^{\mathrm{p}+1}}+\frac{(\mathrm{p}-\alpha)(\mathrm{k}+2 \alpha)}{(\mathrm{k}+\alpha)} \mathrm{r}^{\mathrm{k}-1} \quad(\mathrm{k} \geq 2) \text {. }
$$

for $0<|\mathrm{z}|=\mathrm{r}<1$ and $\mathrm{p} / 2 \leq \alpha<\mathrm{p}$.

For $\mathrm{p}=1$, we get the following corollary.

Corollary 4.3a : If $\mathrm{f}(\mathrm{z}) \in \mathrm{M}_{1}^{\mathrm{k}}(\mathrm{m}, \alpha)$, then

$$
\frac{1}{\mathrm{r}}-\left(\frac{1-\alpha}{\mathrm{k}+\alpha}\right) \mathrm{r}^{\mathrm{k}} \leq\left|\mathrm{D}^{\mathrm{m}} \mathrm{f}(\mathrm{z})\right| \leq \frac{1}{\mathrm{r}}+\left(\frac{1-\alpha}{\mathrm{k}+\alpha}\right) \mathrm{r}^{\mathrm{k}} \quad(\mathrm{k} \geq 1)
$$

and

$$
\frac{1}{\mathrm{r}^{2}}-\frac{(1-\alpha)(\mathrm{k}+2 \alpha)}{(\mathrm{k}+\alpha)} \mathrm{r}^{\mathrm{k}-1} \leq\left|\mathrm{D}^{\mathrm{m}} \mathrm{f}(\mathrm{z})^{\prime}\right| \leq \frac{1}{\mathrm{r}^{2}}+\frac{(1-\alpha)(\mathrm{k}+2 \alpha)}{(\mathrm{k}+\alpha)} \quad(\mathrm{k} \geq 2) .
$$

for $0<|z|=r<1$ and $1 / 2 \leq \alpha<1$.

Corollary 4.3b : If $\mathrm{f}(\mathrm{z}) \in \mathrm{M}_{1}^{\mathrm{k}}(\mathrm{m}, \alpha)$ with $\mathrm{a}_{\mathrm{k}}=0$, then corollary 4.3a holds true for $0<|\mathrm{z}|=\mathrm{r}<1$ and $0 \leq \alpha<1$. for $\alpha=0$ and $\mathrm{m}=0$, we get

Corollary 4.4 : If $\mathrm{f}(\mathrm{z}) \in \mathrm{M}_{\mathrm{p}}^{\mathrm{k}}(0,0) \equiv \mathrm{S}_{\mathrm{p}}^{\mathrm{k}}(0) \equiv \mathrm{S}_{\mathrm{p}}^{\mathrm{k}}$, then

$$
\frac{1}{\mathrm{r}^{\mathrm{p}}}-\frac{\mathrm{p}}{\mathrm{k}} \mathrm{r}^{\mathrm{k}} \leq|\mathrm{f}(\mathrm{z})| \leq \frac{1}{\mathrm{r}^{\mathrm{p}}}+\frac{\mathrm{p}}{\mathrm{k}} \mathrm{r}^{\mathrm{k}} \quad(\mathrm{k} \geq 1)
$$

and

$$
\frac{\mathrm{p}}{\mathrm{r}^{\mathrm{p}+1}}-\mathrm{pr}^{\mathrm{k}-1} \leq\left|\mathrm{f}^{\prime}(\mathrm{z})\right| \leq \frac{\mathrm{p}}{\mathrm{r}^{\mathrm{p}+1}}+\mathrm{pr}^{\mathrm{k}-1} \quad(\mathrm{k} \geq 2)
$$

for $\alpha=0$ and $p=1$, we get the following corollary.

Corollary 4.5a : If $\mathrm{f}(\mathrm{z}) \in \mathrm{M}_{1}^{\mathrm{k}}(\mathrm{m}, 0)$, then

$$
\frac{1}{\mathrm{r}}-\frac{1}{\mathrm{k}} \mathrm{r}^{\mathrm{k}-1} \leq\left|\mathrm{D}^{\mathrm{m}} \mathrm{f}(\mathrm{z})\right| \leq \frac{1}{\mathrm{r}}+\frac{\mathrm{r}^{\mathrm{k}-1}}{\mathrm{k}} \quad(\mathrm{k} \geq 1)
$$$$
\text { and } \quad \frac{1}{\mathrm{r}}-\mathrm{r}^{\mathrm{k}-1} \leq\left|\mathrm{D}^{\mathrm{m}} \mathrm{f}(\mathrm{z})^{\prime}\right| \leq \frac{1}{\mathrm{r}}-\mathrm{r}^{\mathrm{k}-1} \quad(\mathrm{k} \geq 2)
$$

for $0<|z|=r<1$ and $1 / 2 \leq \alpha<1$.

Corollary 4.5b : If $\mathrm{f}(\mathrm{z}) \in \mathrm{M}_{1}^{\mathrm{k}}(\mathrm{m}, 0)$, with $\mathrm{a}_{\mathrm{k}}=0$, then corollary 4.5 a holds true for $0<|\mathrm{z}|=\mathrm{r}<1$ and $0 \leq \alpha<1$. 


\section{CLOSURE THEOREMS :}

Theorem 4.1 : If $\mathrm{f}(\mathrm{z})=\frac{1}{\mathrm{z}^{\mathrm{p}}}+\sum_{\mathrm{n}=0}^{\infty} \mathrm{a}_{\mathrm{n}+\mathrm{k}} \mathrm{z}^{\mathrm{n}+\mathrm{k}} \quad, \quad \mathrm{a}_{\mathrm{n}+\mathrm{k}} \geq 0$

and

$$
g(z)=\frac{1}{z^{p}}+\sum_{n=0}^{\infty} b_{n+k} z^{n+k} \quad, \quad b_{n+k} \geq 0
$$

are in $\mathrm{M}_{\mathrm{p}}^{\mathrm{k}}(\mathrm{m}, \alpha)$, then $\mathrm{h}(\mathrm{z})=\frac{1}{\mathrm{z}^{\mathrm{p}}}+\frac{1}{2} \sum_{\mathrm{n}=0}^{\infty}\left(\mathrm{a}_{\mathrm{n}+\mathrm{k}}+\mathrm{b}_{\mathrm{n}+\mathrm{k}}\right) \mathrm{z}^{\mathrm{n}+\mathrm{k}}$ is also in $\mathrm{M}_{\mathrm{p}}^{\mathrm{k}}(\mathrm{m}, \alpha)$. For $\mathrm{p} / 2 \leq \alpha<\mathrm{p}$.

Proof : $\mathrm{f}(\mathrm{z})$ and $\mathrm{g}(\mathrm{z})$ being in $\mathrm{M}_{\mathrm{p}}^{\mathrm{k}}(\mathrm{m}, \alpha)$, we have

$$
\sum_{n=0}^{\infty}(n+k+\alpha) \frac{(m+n+p+k) !}{m !(n+p+k) !} a_{n+k} \leq(p-\alpha)
$$

(5.2) and

$$
\sum_{n=0}^{\infty}(n+k+\alpha) \frac{(m+n+p+k) !}{m !(n+p+k) !} b_{n+k} \leq(p-\alpha)
$$

for $\mathrm{p} / 2 \leq \alpha<\mathrm{p}$.

It is sufficient for $h(z)$ to be a member of $M_{p}^{k}(m, \alpha)$ to show

$$
\frac{1}{2} \sum_{n=0}^{\infty}(n+k+\alpha) \frac{(m+n+p+k) !}{m !(n+p+k) !}\left(a_{n+k}+b_{n+k}\right) \leq(p-\alpha)
$$

which will follow immediately by use of (5.1) and (5.2).

Theorem 5.2 : The class $M_{\mathrm{p}}^{\mathrm{k}}(\mathrm{m}, \alpha)$ is closed under convex linear combination.

Proof : Let the function $\mathrm{F}_{\mathrm{j}}(\mathrm{z})$ given by

$$
F_{j}(z)=\frac{1}{z^{p}}+\sum_{n=0}^{\infty} a_{n+k, j} z^{n+k} \quad(j=1,2, \ldots), \quad a_{n+k, j} \geq 0
$$

be in $M_{p}^{k}(m, \alpha)$. Then it is enough to show that the function

$$
\mathrm{H}(\mathrm{z})=\lambda \mathrm{F}_{1}(\mathrm{z})+(1-\lambda) \mathrm{F}_{2}(\mathrm{z}) \quad(0 \leq \lambda \leq 1)
$$

is also in $\mathrm{M}_{\mathrm{p}}^{\mathrm{k}}(\mathrm{m}, \alpha)$. For $0 \leq \lambda \leq 1$

$$
H(z)=\frac{1}{z^{p}}+\sum_{n=0}^{\infty}\left(\lambda a_{n+k, 1}+(1-\lambda) a_{n+k, 2}\right) z^{n+k}
$$

we observe that

$$
\begin{aligned}
\sum_{\mathrm{n}=0}^{\infty} \frac{(\mathrm{n}+\mathrm{k}+\alpha)(\mathrm{m}+\mathrm{n}+\mathrm{p}+\mathrm{k}) !}{\mathrm{m} !(\mathrm{n}+\mathrm{p}+\mathrm{k}) !}\left[\lambda \mathrm{a}_{\mathrm{n}+\mathrm{k}, 1}+(1-\lambda) \mathrm{a}_{\mathrm{n}+\mathrm{k}, 2}\right] \\
=\lambda \sum_{\mathrm{n}=0}^{\infty} \frac{(\mathrm{n}+\mathrm{k}+\alpha)(\mathrm{m}+\mathrm{n}+\mathrm{p}+\mathrm{k}) !}{\mathrm{m} !(\mathrm{n}+\mathrm{p}+\mathrm{k}) !} \lambda \mathrm{a}_{\mathrm{n}+\mathrm{k}, 1}+(1-\lambda) \\
\quad \times \sum_{\mathrm{n}=0}^{\infty} \frac{(\mathrm{n}+\mathrm{k}+\alpha)(\mathrm{m}+\mathrm{n}+\mathrm{p}+\mathrm{k}) !}{\mathrm{m} !(\mathrm{n}+\mathrm{p}+\mathrm{k}) !} \mathrm{a}_{\mathrm{n}+\mathrm{k}, 2} \\
\leq \lambda(\mathrm{p}-\alpha)+(1-\lambda)(\mathrm{p}-\alpha) \\
\leq(\mathrm{p}-\alpha)
\end{aligned}
$$

By theorem (3.2), we have $H(z) \in M_{p}^{k}(m, \alpha)$.

Theorem 5.3 : Let $\mathrm{f}_{0}(\mathrm{z})=\frac{1}{\mathrm{z}^{\mathrm{p}}}$ and

$$
\mathrm{f}_{\mathrm{n}+\mathrm{k}}(\mathrm{z})=\frac{1}{\mathrm{z}^{\mathrm{p}}}+\frac{(\mathrm{p}-\alpha) \mathrm{m} !(\mathrm{n}+\mathrm{k}+\mathrm{p}) !}{(\mathrm{m}+\mathrm{p}+\mathrm{n}+\mathrm{k}) !(\mathrm{n}+\mathrm{k}+\alpha)} \mathrm{z}^{\mathrm{n}+\mathrm{k}} \quad, \quad \mathrm{n}=0,1,2 \ldots
$$

Then $\mathrm{f}(\mathrm{z}) \in \mathrm{M}_{\mathrm{p}}^{\mathrm{k}}(\mathrm{m}, \alpha)$ if and only if $\mathrm{f}(\mathrm{z})$ can be expressed in the form 


$$
\mathrm{f}(\mathrm{z})=\sum_{\mathrm{n}=0}^{\infty} \lambda_{\mathrm{n}} \mathrm{f}_{\mathrm{n}}(\mathrm{z}) \quad \lambda_{1}=\lambda_{2}=\ldots=\lambda_{\mathrm{k}-1}=0
$$

where $\sum_{\mathrm{n}=0}^{\infty} \lambda_{\mathrm{n}}=1, \lambda_{\mathrm{n}} \geq 0$ and $\lambda_{\mathrm{n}}=0$ for $\mathrm{n}=1,2, \ldots \mathrm{k}-1 \quad(\mathrm{n}<\mathrm{k}$ and $\mathrm{k} \geq 2)$.

Proof : Suppose that $f(z)=\sum_{n=0}^{\infty} \lambda_{n} f_{n}(z)$ thus,

$$
f(z)=\frac{1}{z^{p}}+\sum_{n=0}^{\infty} \lambda_{n+k}\left\{\frac{(p-\alpha) m !(n+k+p) !}{(m+p+n+k) !(n+k+\alpha)}\right\} z^{n+k}
$$

Since

$$
\begin{aligned}
\sum_{n=0}^{\infty} \frac{(m+p+n+k) !(n+k+\alpha)}{(p-\alpha) m !(n+k+p) !} \lambda_{n+k} \frac{(p-\alpha) m !(n+k+p) !}{(m+p+n+k) !(n+k+\alpha)} & =\sum_{n=0}^{\infty} \lambda_{n+k} \\
& =\sum_{n=1}^{\infty} \lambda_{n} \\
& =1-\lambda_{0} \leq 1
\end{aligned}
$$

Thus, by theorem (3.2) $f(z) \in M_{p}^{k}(m, \alpha)$.

Conversely : Suppose that the function $f(z)$ defined by (1.1) belongs to $M_{p}^{k}(m, \alpha)$, then by theorem (3.2), we have

$$
a_{n+k} \leq \frac{(p-\alpha) m !(n+k+p) !}{(m+p+n+k) !(n+k+\alpha)} \quad, n \geq 0 .
$$

Setting $\quad \lambda_{n+k}=\frac{(m+p+n+k) !(n+k+\alpha) a_{n+k}}{(p-\alpha) m !(n+k+p) !}$

with $\quad \lambda_{1}=\lambda_{2}=\ldots=\lambda_{\mathrm{k}-1}=0 \quad$ and $\sum_{\mathrm{n}=0}^{\infty} \lambda_{\mathrm{n}}=1, \lambda_{\mathrm{n}} \geq 0$

then

$$
\begin{aligned}
f(z) & =\frac{1}{z^{p}}+\sum_{n=0}^{\infty} a_{n+k} z^{n+k} \\
& =\frac{1}{z^{p}}+\sum_{n=0}^{\infty} \frac{(p-\alpha) m !(n+k+p) !}{(m+p+n+k) !(n+k+\alpha)} \lambda_{n+k} z^{n+k} \\
& =\frac{1}{z^{p}}+\sum_{n=0}^{\infty} \lambda_{n+k}\left\{f_{n+k}-\frac{1}{z^{p}}\right\} \\
& =\frac{1}{z^{p}}+\left(1-\sum_{n=0}^{\infty} \lambda_{n+k}\right)+\sum_{n=0}^{\infty} \lambda_{n+k} f_{n+k} \\
& =\left(1-\sum_{n=1}^{\infty} \lambda_{n}\right) \frac{1}{z^{p}}+\sum_{n=1}^{\infty} \lambda_{n} f_{n} \\
& =\lambda_{0} f_{0}+\sum_{n=1}^{\infty} \lambda_{n} f_{n} \\
& =\sum_{n=0}^{\infty} \lambda_{n} f_{n}(z)
\end{aligned}
$$

This completes the proof. 


\section{RADII OF STARLIKENESS AND CONVEXITY :}

Theorem 6.1 : Let the function $f(z)$ defi0ned by (1.1) be in the class $M_{p}^{k}(m, \alpha)$, then $f(z)$ is meromorphically $\mathrm{p}$-valent starlike of order $\delta(\mathrm{p} / 2 \leq \delta<\mathrm{p})$ in the disk $|\mathrm{z}|<\mathrm{r}_{1}$, where

$$
\mathrm{r}_{1}=\mathrm{r}_{1}(\alpha, \delta)=\inf _{\mathrm{n}}\left[\frac{(\mathrm{n}+\mathrm{k}+\alpha)(\mathrm{m}+\mathrm{p}+\mathrm{n}+\mathrm{k}) !(\mathrm{p}-\delta)}{(\mathrm{n}+\mathrm{k}+\delta) \mathrm{m} !(\mathrm{n}+\mathrm{p}+\mathrm{k}) !(\mathrm{p}-\alpha)}\right]^{\frac{1}{\mathrm{n}+\mathrm{k}+\mathrm{p}}} \quad \mathrm{n} \in \mathrm{N}_{0}
$$

Proof : It is sufficient to prove that

$$
\left|\frac{\mathrm{zf}^{\prime}(\mathrm{z})+\mathrm{pf}(\mathrm{z})}{z f^{\prime}(\mathrm{z})+(2 \delta-\mathrm{p}) \mathrm{f}(\mathrm{z})}\right| \leq 1 \quad \text { for } 0<|\mathrm{z}|<\mathrm{r}_{1}
$$

we have

$$
\begin{aligned}
\left|\frac{z^{\prime}(z)+p f(z)}{z f^{\prime}(z)+(2 \delta-p) f(z)}\right| & =\left|\frac{\sum_{n=0}^{\infty}(n+k+p) a_{n+k} z^{n+k}}{\frac{2(\delta-p)}{z^{p}}+\sum_{n=0}^{\infty}(n+k+2 \delta-p) a_{n+k} z^{n+k}}\right| \\
& =\left|\frac{\sum_{n=0}^{\infty}(n+k+p) a_{n+k} z^{n+k+p}}{-2(p-\delta)+\sum_{n=0}^{\infty}(n+k+2 \delta-p) a_{n+k} z^{n+k+p}}\right|
\end{aligned}
$$

Thus, the result follows if

$$
\left|\frac{\sum_{n=0}^{\infty}(n+k+p) a_{n+k} z^{n+k+p}}{-2(p-\delta)+\sum_{n=0}^{\infty}(n+k+2 \delta-p) a_{n+k} z^{n+k+p}}\right| \leq 1
$$

or $\quad \sum_{n=0}^{\infty}(n+k+p) a_{n+k}|z|^{n+k+p} \leq 2(p-\delta)-\sum_{n=0}^{\infty}(n+k+2 \delta-p) a_{n+k}|z|^{n+k+p}$

which is equivalent to

$$
\sum_{n=0}^{\infty}(\mathrm{n}+\mathrm{k}+\delta) \mathrm{a}_{\mathrm{n}+\mathrm{k}}|\mathrm{z}|^{\mathrm{n}+\mathrm{k}+\mathrm{p}} \leq(\mathrm{p}-\delta)
$$

or

$$
\sum_{\mathrm{n}=0}^{\infty} \frac{(\mathrm{n}+\mathrm{k}+\delta)}{(\mathrm{p}-\delta)} \mathrm{a}_{\mathrm{n}+\mathrm{k}}|\mathrm{z}|^{\mathrm{n}+\mathrm{k}+\mathrm{p}} \leq 1
$$

But, by theorem 3.2, we have

$$
\sum_{n=0}^{\infty} \frac{(n+k+\alpha)(m+p+n+k) !}{m !(n+k+p) !(p-\alpha)} a_{n+k} \leq 1
$$

Hence, (6.2) holds if and only if for all $\mathrm{n} \in \mathrm{N}_{0}$

or

$$
\begin{aligned}
& \frac{(\mathrm{n}+\mathrm{k}+\delta)}{(\mathrm{p}-\delta)} \mathrm{a}_{\mathrm{n}+\mathrm{k}}|\mathrm{z}|^{\mathrm{n}+\mathrm{k}+\mathrm{p}} \leq \frac{(\mathrm{n}+\mathrm{k}+\alpha)(\mathrm{m}+\mathrm{p}+\mathrm{n}+\mathrm{k}) !}{\mathrm{m} !(\mathrm{n}+\mathrm{k}+\mathrm{p}) !(\mathrm{p}-\alpha)} \mathrm{a}_{\mathrm{n}+\mathrm{k}} \\
& |\mathrm{z}|^{\mathrm{n}+\mathrm{k}+\mathrm{p}} \leq \frac{(\mathrm{n}+\mathrm{k}+\alpha)(\mathrm{m}+\mathrm{p}+\mathrm{n}+\mathrm{k}) !(\mathrm{p}-\delta)}{(\mathrm{n}+\mathrm{k}+\delta) \mathrm{m} !(\mathrm{n}+\mathrm{k}+\mathrm{p}) !(\mathrm{p}-\alpha)} \\
& |\mathrm{z}| \leq\left[\frac{(\mathrm{n}+\mathrm{k}+\alpha)(\mathrm{m}+\mathrm{p}+\mathrm{n}+\mathrm{k}) !(\mathrm{p}-\delta)}{(\mathrm{n}+\mathrm{k}+\delta) \mathrm{m} !(\mathrm{n}+\mathrm{p}+\mathrm{k}) !(\mathrm{p}-\alpha)}\right]^{\frac{1}{\mathrm{n}+\mathrm{k}+\mathrm{p}}}
\end{aligned}
$$

This completes the theorem. 
Theorem 6.2 : Let the function $\mathrm{f}(\mathrm{z})$ defined by $(1.1)$ be in the class $\mathrm{M}_{\mathrm{p}}^{\mathrm{k}}(\mathrm{m}, \alpha)$. Then $\mathrm{f}(\mathrm{z})$ is meromorphically $\mathrm{p}-$ valent convex of order $\delta(\mathrm{p} / 2 \leq \delta<\mathrm{p})$ in the disk $|\mathrm{z}|<\mathrm{r}_{2}$ where $\mathrm{r}_{2}=\mathrm{r}_{2}(\alpha, \delta)$

$$
=\inf _{n}\left[\frac{(n+k+\alpha)(m+p+n+k) !(p-\delta) p}{(n+k)(n+k+\delta) m !(n+p+k) !(p-\alpha)}\right]^{\frac{1}{n+k+p}} \quad n \in N_{0}
$$

Theorem 6.3: Let function $f(z)$ given by (1.1) be in the class $M_{p}^{k}(m, \alpha)$.

$$
\mathrm{F}(\mathrm{z})=\frac{1}{\mathrm{c}}\left[(\mathrm{c}+\mathrm{p}) \mathrm{f}(\mathrm{z})+\mathrm{zf}^{1}(\mathrm{z})\right], \mathrm{c}>0
$$

Then, $\mathrm{F}(\mathrm{z})$ is in $\mathrm{M}_{\mathrm{p}}^{\mathrm{k}}(\mathrm{m}, \alpha)$ for $|\mathrm{z}| \leq \mathrm{r}(\alpha, \beta)$, where

$$
r(\alpha, \beta)=\inf _{n}\left[\frac{(n+k+\alpha)(p-\beta) c}{(n+k+\beta)(c+p+n+k)(p-\alpha)}\right]^{\frac{1}{n+k+p}} \quad, n=0,1,2 \ldots
$$

The result is sharp for the function $\mathrm{f}_{\mathrm{n}}(\mathrm{z})$ given by (3.6).

Proof : $F(z)=\frac{1}{z^{p}}+\sum_{n=0}^{\infty} \frac{(c+p+n+k)}{c} a_{n+k} z^{n+k}$

Let $\quad w(z)=1-\frac{(m+1)}{(p-\beta)}\left\{\frac{D^{m+1} F(z)}{D^{m} F(z)}-1\right\}$

Then it is sufficient to show that

$$
\left|\frac{\mathrm{w}(\mathrm{z})-1}{\mathrm{w}(\mathrm{z})+1}\right|<1
$$

A computation shows that this is satisfied if

$$
\sum_{n=0}^{\infty} \frac{(n+k+\beta)(m+p+n+k) !(c+p+n+k)}{(p-\beta) m !(n+k+p) ! c} a_{n+k}|z|^{n+p+k} \leq 1
$$

Since $f(z) \in M_{p}^{k}(m, \alpha)$, by theorem 3.2, we have

$$
\sum_{n=0}^{\infty} \frac{(n+k+\alpha)(m+p+n+k) !}{(p-\alpha) m !(n+k+p) !} a_{n+k} \leq 1
$$

The equation (6.3) is satisfied if

$$
\begin{gathered}
\sum_{n=0}^{\infty} \frac{(\mathrm{n}+\mathrm{k}+\beta)(\mathrm{m}+\mathrm{p}+\mathrm{n}+\mathrm{k}) !(\mathrm{c}+\mathrm{p}+\mathrm{n}+\mathrm{k})}{(\mathrm{p}-\beta) \mathrm{m} !(\mathrm{n}+\mathrm{k}+\mathrm{p}) ! \mathrm{c}}|\mathrm{z}|^{\mathrm{n}+\mathrm{p}+\mathrm{k}} \mathrm{a}_{\mathrm{n}+\mathrm{k}} \\
\leq \sum_{\mathrm{n}=0}^{\infty} \frac{(\mathrm{n}+\mathrm{k}+\alpha)(\mathrm{m}+\mathrm{p}+\mathrm{n}+\mathrm{k}) !}{(\mathrm{p}-\alpha) \mathrm{m} !(\mathrm{n}+\mathrm{k}+\mathrm{p}) !} \mathrm{a}_{\mathrm{n}+\mathrm{k}}
\end{gathered}
$$

or

$$
|z| \leq\left[\frac{(n+k+\alpha)(p-\beta) c}{(n+k+\beta)(c+p+n+k)(p-\alpha)}\right]^{\frac{1}{n+k+p}}
$$

\section{INTEGRAL OPERATOR}

Theorem 7.1 : Let the function $f(z)$ given by (1.1) be in $M_{p}^{k}(m, \alpha)$. Then the integral operator

$$
\begin{aligned}
\mathrm{F}(\mathrm{z})=(\mathrm{c}-\mathrm{p}+1) \int_{0}^{1} \mathrm{u}^{\mathrm{c}} \mathrm{f}(\mathrm{uz}) \mathrm{du} & \text { for } \mathrm{p} \leq \mathrm{c}<\infty \\
0<\mathrm{u} & \leq 1
\end{aligned}
$$

is in $\mathbf{M}_{\mathrm{p}}^{\mathrm{k}}(\mathrm{m}, \delta)$ where 


$$
\delta=\frac{\mathrm{p}(\mathrm{p}+\alpha)(\mathrm{c}+\mathrm{k}+1)-\mathrm{p}(\mathrm{p}-\alpha)(\mathrm{c}-\mathrm{p}+1)}{(\mathrm{c}-\mathrm{p}+1)(\mathrm{p}-\alpha)+(\mathrm{p}+\alpha)(\mathrm{c}+\mathrm{k}+1)}
$$

This result is sharp for the function

$$
f(z)=\frac{1}{z^{p}}+\frac{(p-\alpha) m !(k+p) !}{(k+\alpha)(m+p+k) !} z^{n+k}
$$

Proof : Let $\mathrm{f}(\mathrm{z}) \in \mathrm{M}_{\mathrm{p}}^{\mathrm{k}}(\mathrm{m}, \alpha)$. Then

$$
\begin{aligned}
F(z)=(c-p+1) & \int_{0}^{1} u^{c} f(u z) d u \\
= & (c-p+1) \int_{0}^{1}\left(\frac{u^{c-p}}{z^{p}}+\sum_{n=0}^{\infty} a_{n+k} u^{c+n+k} z^{n+k}\right) d u \\
= & \frac{1}{z^{p}}+\sum_{n=0}^{\infty} \frac{c-p+1}{c+n+k+1} a_{n+k} z^{n+k}
\end{aligned}
$$

It is sufficient to show that

$$
\sum_{n=0}^{\infty} \frac{(c-p+1)(m+n+p+k) !(n+p+\delta)}{(c+n+k+1)(p-\delta)(n+k+p) ! m !} a_{n+k} \leq 1
$$

Since $f(z) \in M_{p}^{k}(m, \alpha)$, we have

(7.1) is satisfied if

$$
\sum_{n=0}^{\infty} \frac{(m+n+p+k) !(n+p+\alpha)}{m !(n+k+p) !(p-\alpha)} \leq 1
$$

or

$$
(\mathrm{c}-\mathrm{p}+1)(\mathrm{n}+\mathrm{p}+\delta)(\mathrm{p}-\alpha) \leq(\mathrm{c}+\mathrm{n}+\mathrm{k}+1)(\mathrm{p}-\delta)(\mathrm{n}+\mathrm{p}+\alpha)
$$

A computation shows that

$$
\delta \leq \frac{\mathrm{p}(\mathrm{c}+\mathrm{n}+\mathrm{k}+1)(\mathrm{n}+\mathrm{p}+\alpha)-(\mathrm{c}-\mathrm{p}+1)(\mathrm{n}+\mathrm{p})(\mathrm{p}-\alpha)}{(\mathrm{c}-\mathrm{p}+1)(\mathrm{p}-\alpha)+(\mathrm{c}+\mathrm{n}+\mathrm{k}+1)(\mathrm{n}+\mathrm{p}+\alpha)}=\mathrm{F}(\mathrm{n})
$$

$\mathrm{F}(\mathrm{n}+1)-\mathrm{F}(\mathrm{n})$

$$
=\frac{(p-\alpha)(c-p+1)[(n+p)(n+2 p+1)+p(\alpha+p+1)+p n+k(p-\alpha)]}{[(c-p+1)(p-\alpha)+(c+n+k+2)(n+1+p+\alpha)][(c-p+1)(p-\alpha)+(c+n+k+1)(n+p+\alpha)]}>0
$$

for all $n \geq 0$. This means $F(n)$ is increasing and $F(n) \geq F(0)$. Using this the result follows.

\section{NEIGHBOURHOODS FOR THE CLASS $M_{p}^{k}(m, \alpha, \gamma)$ :}

Definition 8.1 : A function $\mathrm{f}(\mathrm{z}) \in \mathrm{M}_{\mathrm{p}}^{\mathrm{k}}$ is said to be in the class $\mathrm{M}_{\mathrm{p}}^{\mathrm{k}}(\mathrm{m}, \alpha, \gamma)$ if there exist a function $\mathrm{g}(\mathrm{z}) \in$ $\mathrm{M}_{\mathrm{p}}^{\mathrm{k}}(\mathrm{m}, \alpha)$ such that

$$
\left|\frac{\mathrm{f}(\mathrm{z})}{\mathrm{g}(\mathrm{z})}-1\right| \leq 1-\gamma \quad, \quad 0 \leq \gamma<1 .
$$

Definition 8.2 : $\mathrm{N}_{\delta}(\mathrm{f})$ denote the $\delta$-neighbourhood of the function $\mathrm{f} \in \mathrm{M}_{\mathrm{p}}^{\mathrm{k}}$ of the form (1.1), i.e.

$$
\begin{array}{r}
N_{\delta}(f)=\left\{g \in M_{p}^{k}: g(z)=\frac{1}{z^{p}}+\sum_{n=0}^{\infty} b_{n+k} z^{n+k}\right. \\
\text { and } \left.\sum_{n=0}^{\infty}(n+k)\left|a_{n+k}-b_{n+k}\right| \leq \delta\right\}
\end{array}
$$

Theorem 8.1 : If $g(z) \in M_{p}^{k}(m, \alpha)$ and 


$$
\frac{\delta(\mathrm{k}+\alpha)(\mathrm{m}+\mathrm{k}+\mathrm{p}) !}{(\mathrm{m}+\mathrm{k}+\mathrm{p}) !-\mathrm{m} !(\mathrm{p}-\alpha)(\mathrm{k}+\mathrm{p}) !]}
$$

Then $\mathrm{N}_{\delta}(\mathrm{g}) \subset \mathrm{M}_{\mathrm{p}}^{\mathrm{k}}(\mathrm{m}, \alpha, \gamma)$.

Proof : Let $\mathrm{f}(\mathrm{z}) \in \mathrm{N}_{\delta}(\mathrm{g})$, then we find from (8.2) that

$$
\sum_{n=0}^{\infty}\left|a_{n+k}-b_{n+k}\right| \leq \frac{\delta}{k}
$$

Since $g(z) \in M_{p}^{k}(m, \alpha)$, we have from (3.3)

$$
\begin{aligned}
\sum_{n=0}^{\infty} b_{n+k} & \leq \frac{(p-\alpha) m !(k+p) !}{(k+\alpha)(m+p+k) !} \\
\left|\frac{f(z)}{g(z)}-1\right| & =\mid \frac{\sum_{n=0}^{\infty}\left(a_{n+k}-b_{n+k}\right) \mid}{1+\sum_{n=0}^{\infty} b_{n+k} \mid} \\
& \leq \frac{1-\sum_{n=0}^{\infty} b_{n+k}}{\left|a_{n+k}-b_{n+k}\right|} \\
& =\frac{k}{k\left[1-\frac{(p-\alpha) m !(k+p) !}{(k+\alpha)(m+k+p) !} \quad(u s i n g(8.4) \&\right.} \\
& =\frac{\delta(k+\alpha)(m+k+p) !}{k[(k-\alpha)(m+k+p) !-(p-\alpha) m !(k+p) !]} \\
& =1-\gamma \quad
\end{aligned}
$$

where $\gamma$ is given by (8.3). Hence by definition $8.1 \mathrm{f}(\mathrm{z}) \in \mathrm{M}_{\mathrm{p}}^{\mathrm{k}}(\mathrm{m}, \alpha, \gamma)$ which completes the proof.

\section{References}

[1]. M.K. AOUF and H.M. SRIVASTAVA, A new criterion for meromorphically p-valent convex functions of order $\alpha$, Math.Sci.Res. Hot. Line 1(8)(1997), 7-12.

[2]. S.B. JOSHI and H.M. SRIVASTAVA, A certain family of meromorphically multivalent functions, Computers Math. App. 38(3/4) (1999), 201-211.

[3]. J.L. LIU and H.M. SRIVASTAVA, A linear operator and associated families of meromorphically multivalent functions. J. Math. Anal. Appl. 259(2001), 566-581.

[4]. J.L. LIU and S. OWA, On a class of meromorphic p-valent functions involving certain linear operators. Intermat. J. Math. Math. Sci. 32(2002), 271-280.

[5]. J.L. LIU and H.M. SRIVASTAVA, Some convolution conditions for starlikeness and convexity of meromorphically multivalent functions, Applied Math. Letters, 16(2003), 13-16.

[6]. S. OWA, H.E. DARWISH and M.K. AOUF, Meromorphic multivalent functions with positive and fixed second coefficients, Math. Japan, 46(1997), 231-236.

[7]. H.M. SRIVASTAVA, H.M. HOSSEN and M.K. AOUF, A unified presentation of some classes of meromorphically multivalent functions, Computers Math. Appl. 38(11/12)(1999), 63-70. 